\title{
Correlation Between Influenza-Like IIIness Reported by ILINet and NSSP, Kansas, 2014-2015
}

\author{
Daniel J. Neises* and Farah Naz \\ Bureau of Epidemiology and Public Health Informatics, Kansas Department of Health and Environment, Topeka, KS, USA
}

\section{Objective}

Measure the correlation between Influenza-like Illness (ILI) data collected by the U.S. Outpatient Influenza-like Illness Surveillance Network (ILINet) and the National Syndromic Surveillance Program (NSSP) in Kansas for the 2014-2015 influenza surveillance period.

\section{Introduction}

Influenza is not a notifiable disease in Kansas; patient-level influenza data is not reported to the Kansas Department of Health and Environment (KDHE). Kansas' primary method of influenza surveillance is the U.S. Outpatient Influenza-like Illness Surveillance Network (ILINet), a collaboration between the Centers for Disease Control and Prevention (CDC) and state health departments. During the 2014-2015 influenza surveillance period (September 28, 2014 through May 16, 2015), 35 health care providers (20 family practice clinics, nine hospital emergency departments, four university student health centers, and two pediatric clinics) served as ILINet sites. Providers were instructed to report the previous week's influenzalike illness (ILI) data, including the number of patients who met the ILI case definition and the total number of patients seen, by 11:00 AM each Tuesday. An average of 16 providers (45\%) met the deadline each week.

Kansas' method of syndromic surveillance is the National Syndromic Surveillance Program (NSSP). During the 2014-2015 surveillance period, 98 of 129 Kansas hospital emergency departments (EDs) automated submission of electronic health record data to NSSP. Twenty-three EDs submitted data at least once per day throughout the season; the remaining EDs were still undergoing testing and validation to meet minimum data quality standards, and therefore were prone to erratic data submission and indeterminate data quality.

\section{Methods}

The weekly proportion of ILINet provider patients who met the ILINet ILI case definition (Fever $\geq 100^{\circ} \mathrm{F}$ with cough or sore throat) were compared to the weekly proportion of NSSP-enrolled emergency department patients who met the NSSP Syndrome Definitions Work Group ILI syndrome definition (ILI-S). Patients were included in ILI-S if influenza-related symptoms or diagnosis codes were present in the chief complaint, diagnosis code, or diagnosis text portions of their NSSP record.

ILINet providers that also submitted data to NSSP were removed from the ILINet data set to ensure those seven emergency departments did not influence correlation.

ILINet and NSSP data submitted before the weekly ILINet deadline were compared to evaluate if NSSP could be used as a proxy for accurate situational awareness in Kansas, given NSSP's automatic, daily data submission and the reporting delays seen with ILINet.

Pearson correlation coefficients (rho) were calculated using SAS 9.3 (SAS Institute Inc., Cary, NC, USA)

\section{Results}

ILINet and NSSP ILI data were highly correlated, both when comparing all data submitted during the surveillance period $($ rho $=0.91, \mathrm{P}<0.001$; Figure 1$)$ and data submitted prior to the weekly ILINet deadline (rho=0.69, $\mathrm{P}<0.001$; Figure 2 ).

\section{Conclusions}

Despite the differences in ILI definitions applied to each surveillance system, variation in the number of sites submitting data each week to each system, and differences in the type, geographic distribution, and total number of reporting sites, the weekly proportions of ILI patients reported to ILINet and NSSP were highly correlated. The correlation was higher when comparing all data collected during the surveillance period than when comparing only data submitted before the weekly ILINet deadline. Applying the ILI-S syndrome definition to NSSP data may provide useful situational awareness for states whose ILINet providers do not routinely meet the weekly data submission deadline.

Figure 1: Proportion of Patients Meeting the Influenzalike Illness Definition by Surveillance System, Kansas, 2014-2015 Influenza Surveillance Period

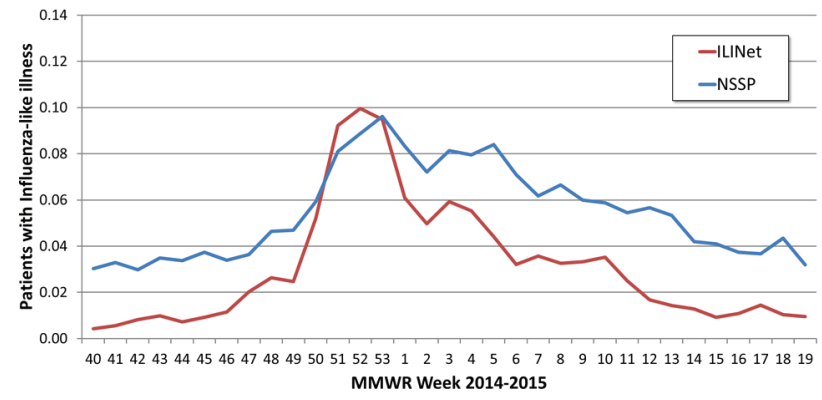

Figure 2: Proportion of Patients Meeting the Influenzalike Illness Definition as Reported at 11:00 A.M. every Tuesday by Surveillance System, Kansas, 2014-2015 Influenza Surveillance Period

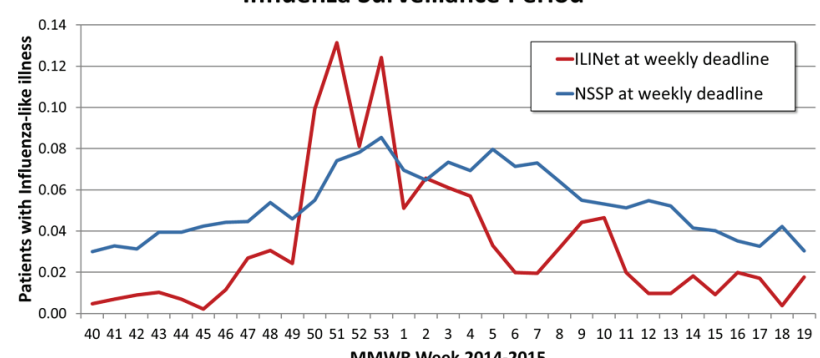

MMWR Week 2014-2015

\section{Keywords}

Influenza like illness; Syndromic surveillance; NSSP; ILINet; Influenza

\section{Acknowledgments}

The authors wish to thank CDC's Influenza Division and the NSSP Syndrome Definitions Workgroup for their assistance.

\section{*Daniel J. Neises}

E-mail: dneises@kdheks.gov 